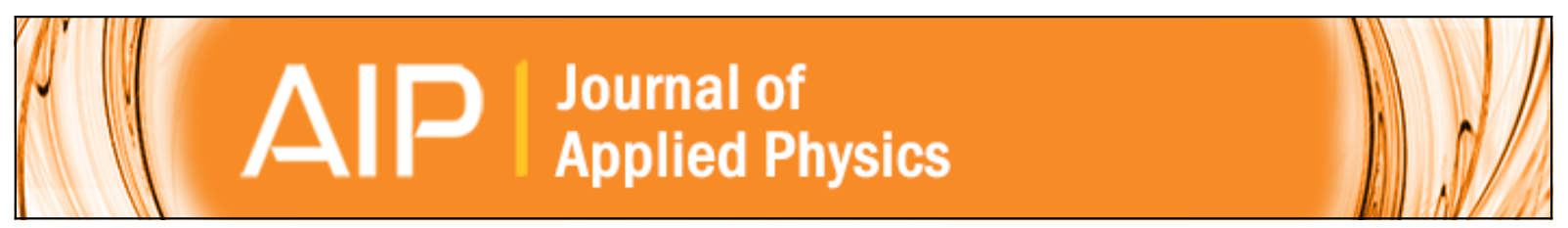

\title{
Exploring SiSn as a performance enhancing semiconductor: A theoretical and experimental approach
}

Aftab M. Hussain, Nirpendra Singh, Hossain Fahad, Kelly Rader, Udo Schwingenschlögl, and Muhammad Hussain

Citation: Journal of Applied Physics 116, 224506 (2014); doi: 10.1063/1.4904056

View online: http://dx.doi.org/10.1063/1.4904056

View Table of Contents: http://scitation.aip.org/content/aip/journal/jap/116/22?ver=pdfcov

Published by the AIP Publishing

\section{Articles you may be interested in}

Phonon lifetime in SiSn and its suitability for hot-carrier solar cells

Appl. Phys. Lett. 104, 222106 (2014); 10.1063/1.4881938

Slater-Pauling behavior in LiMgPdSn-type multifunctional quaternary Heusler materials: Half-metallicity, spingapless and magnetic semiconductors

J. Appl. Phys. 113, 193903 (2013); 10.1063/1.4805063

Low temperature chemical vapor deposition of Si-based compounds via Si H 3 Si H 2 Si H 3 : Metastable Si Sn/ $\mathrm{Ge} \mathrm{Sn/Si} \mathrm{(} 100$ ) heteroepitaxial structures

Appl. Phys. Lett. 89, 231924 (2006); 10.1063/1.2403903

The giant magnetocaloric effect of Gd 5 Si 1.95 Ge 2.05 enhanced by Sn doping

J. Appl. Phys. 100, 073904 (2006); 10.1063/1.2355430

Magnetic and half-metallic properties of the full-Heusler alloys Co $2 \mathrm{Ti} X(\mathrm{X}=\mathrm{Al}, \mathrm{Ga} ; \mathrm{Si}, \mathrm{Ge}, \mathrm{Sn}$; Sb )

J. Appl. Phys. 97, 10C307 (2005); 10.1063/1.1853899

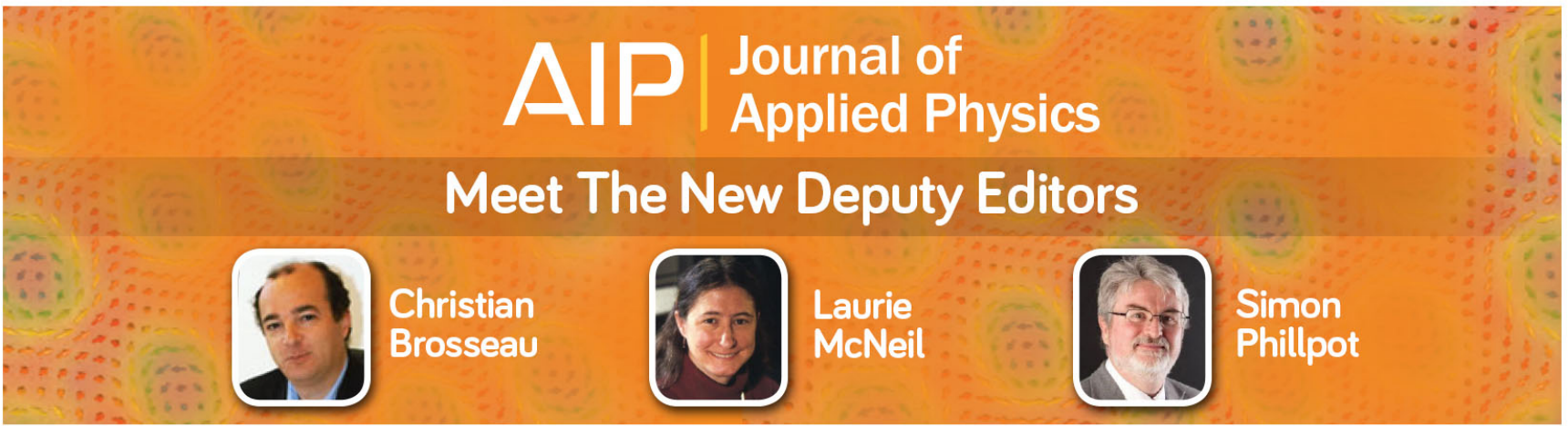




\title{
Exploring SiSn as a performance enhancing semiconductor: A theoretical and experimental approach
}

\author{
Aftab M. Hussain, ${ }^{1}$ Nirpendra Singh, ${ }^{2}$ Hossain Fahad, ${ }^{1}$ Kelly Rader, ${ }^{1}$ \\ Udo Schwingenschlögl, ${ }^{2}$ and Muhammad Hussain ${ }^{1, a)}$ \\ ${ }_{1}^{1}$ Integrated Nanotechnology Lab, King Abdullah University of Science and Technology, Thuwal, Saudi Arabia \\ ${ }^{2}$ Material Science and Engineering Division, King Abdullah University of Science and Technology, Thuwal, \\ Saudi Arabia
}

(Received 6 November 2014; accepted 29 November 2014; published online 11 December 2014)

\begin{abstract}
We present a novel semiconducting alloy, silicon-tin ( $\mathrm{SiSn})$, as channel material for complementary metal oxide semiconductor (CMOS) circuit applications. The material has been studied theoretically using first principles analysis as well as experimentally by fabricating MOSFETs. Our study suggests that the alloy offers interesting possibilities in the realm of silicon band gap tuning. We have explored diffusion of tin ( $\mathrm{Sn}$ ) into the industry's most widely used substrate, silicon (100), as it is the most cost effective, scalable and CMOS compatible way of obtaining SiSn. Our theoretical model predicts a higher mobility for p-channel SiSn MOSFETs, due to a lower effective mass of the holes, which has been experimentally validated using the fabricated MOSFETs. We report an increase of $13.6 \%$ in the average field effect hole mobility for SiSn devices compared to silicon control devices. (C) 2014 AIP Publishing LLC. [http://dx.doi.org/10.1063/1.4904056]
\end{abstract}

\section{INTRODUCTION}

There has been a growing interest in non-silicon semiconductors as potential replacement for silicon based electronics for sustained continuation of Moore's Law. These developments are underway since scaling alone cannot achieve this goal. In particular, short channel effects and high static power consumption due to increased gate leakage hamper scaling efforts. ${ }^{1}$ These challenges can be mitigated by novel channel materials with better charge transport properties. $^{2}$ Prominent among the alternative channel materials being studied are graphene and other single atomic layer materials, ${ }^{3-5}$ III-V semiconductors, ${ }^{6-8}$ oxide semiconductors, ${ }^{9}$ and carbon nanotubes. ${ }^{10}$ Even in case of novel channel materials, a silicon wafer is still used as the starting substrate because of ease of processing and low cost. However, integration of alternative channel materials onto the silicon platform for CMOS fabrication is challenging.

To overcome this challenge, group IVa alloys, which are similar in structure and properties to silicon, are being studied as channel materials. These are alloys of the elements in the group IVa of the periodic table-carbon (C), silicon $(\mathrm{Si})$, germanium $(\mathrm{Ge})$, tin $(\mathrm{Sn})$, and lead $(\mathrm{Pb})$. Their similar lattice structure and properties can make their integration onto a silicon wafer possible. The band structure and semiconducting nature of group IV alloys such as SiGe, SiSn and GeSn have been studied in the past by theoretical analyses of the conduction band and valence band for various compositions. ${ }^{11-13}$ Several works have been dedicated to the fabrication of MOSFETs SiGe and GeSn. ${ }^{14-18}$ In these studies, the semiconducting alloy was grown by either gas phase epitaxy or molecular beam epitaxy, which increases the cost of production significantly.

\footnotetext{
a) Author to whom correspondence should be addressed. Electronic mail: muhammadmustafa.hussain@kaust.edu.sa
}

In this work, we have studied SiSn as a potential channel material using theoretical analysis as well as by fabrication and characterization of MOSFET devices. In our previous works, we have reported SiSn MugFETs obtained using SOI as a starting substrate, ${ }^{19}$ the behavior of SiSn Metal Oxide Semiconductor Capacitors (MOSCAPs), ${ }^{20}$ and the potential application of SiSn MOSFETs for low power circuits. ${ }^{21}$ In this work, we obtain the SiSn channel material using a low cost process of diffusion of Sn atoms into bulk silicon (100) wafers. We have calculated the change in the effective mass of holes as a result of $\mathrm{Sn}$ incorporation into silicon lattice, and the experimental field effect mobility for SiSn transistors.

\section{THEORETICAL ANALYSIS}

The possible effect of having tin ( $\mathrm{Sn})$ at certain silicon lattice positions was first analyzed using first principles study of the alloy. To obtain the band gap correctly, we used the Tran-Blaha modified Becke-Johnson exchange potential, which is computationally economical and avoids atom dependent parameters. ${ }^{22,23}$ We started with supercells of various sizes to obtain $\mathrm{Sn}$ concentration of $1.5 \%, 3 \%$ and $6 \%$. The results show a continuous decrease in the band gap of the alloy with increase in Sn concentration (Table I). In order to analyze the impact of the change in electronic band

TABLE I. Change in band gap with Sn concentration in silicon lattice.

\begin{tabular}{lcc}
\hline \hline Sn concentration $(\%)$ & ${\text { Band } \text { Gap }^{\mathrm{a}}(\mathrm{eV})}$ & ${\text { Band } \text { Gap }^{\mathrm{b}}(\mathrm{eV})}$ \\
\hline 0 & 1.16 & $\ldots$ \\
1.5 & 1.13 & $\ldots$ \\
3 & 1.10 & 1.101 \\
6 & 1.04 & 1.043 \\
\hline
\end{tabular}

${ }^{\mathrm{a}}$ Calculated from first principles anaylsis.

${ }^{\mathrm{b}}$ Calculated using Vegard's Law. 


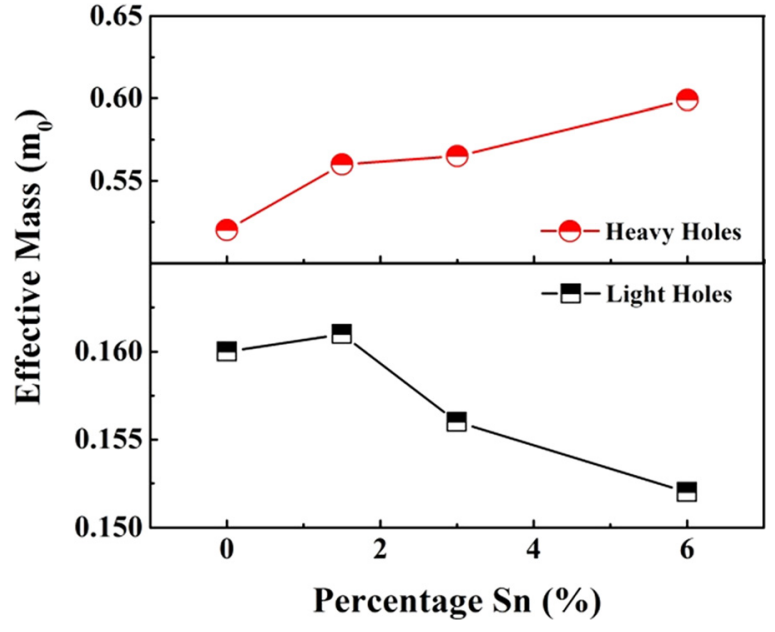

FIG. 1. Change in effective mass of the holes with concentration of Sn in SiSn.

structures on channel conductance, we calculated the effective mass of holes at the edge of the valence band. The effective mass of the holes was obtained from the curvature of each band at extreme $\Gamma$ point.

The change in effective masses of the holes with concentration of $\mathrm{Sn}$ in the alloy is shown in Figure 1. The change in band gap for an alloy can be predicted using the Vegard's law. ${ }^{24}$ We used the value of band gap for $1.5 \%$ Sn concentration to obtain the bowing parameter for Vegard's law. We have reported the bowing parameter to be $0.852 \mathrm{eV}$ in our previous work. ${ }^{19}$ This result was used to calculate the band gap for $3 \%$ and $6 \%$ concentrations using Vegard's law, and it was found that the calculated values closely agreed with the simulation results (Table I). In general, the value of the bowing parameter can vary with composition. To counter this effect and obtain a better fit for the value of the bowing parameter and band gaps subsequently calculated, we determined the bowing parameter for all values of Sn concentrations. We report the calculated values to be $0.852 \mathrm{eV}, 0.866 \mathrm{eV}$, and $0.894 \mathrm{eV}$ for $1.5 \%, 3 \%$, and $6 \% \mathrm{Sn}$ concentration, respectively. Hence, the average bowing parameter is $0.87 \pm 0.02 \mathrm{eV}$.

\section{MOSFET FABRICATION}

It was observed that the effective mass of the light holes is lower for the strained lattice of SiSn. Hence, an improvement in the hole mobility was expected for $\mathrm{SiSn}$ channel MOSFETs. ${ }^{25}$ For experimental verification of the theory, we fabricated p-channel MOSFETs using SiSn as channel material, for which it was essential to obtain good quality mono-crystalline SiSn. We used the thermal diffusion of Sn into the silicon lattice to obtain crystalline SiSn, employing 4 in., n-type, lightly doped (11-20 $\Omega \cdot \mathrm{cm})$ silicon (100) substrate as the starting point for obtaining device quality $\mathrm{SiSn}$. We cleaned the substrate to remove contaminations and native oxides using piranha solution (1:1:3 $\mathrm{H}_{2} \mathrm{SO}_{4}: \mathrm{H}_{2} \mathrm{O}_{2}: \mathrm{H}_{2} \mathrm{O}$ at $120^{\circ} \mathrm{C}$ ) for 10 min and buffered oxide etchant (BOE) for $10 \mathrm{~min}$, respectively. We then deposited a nano-scale layer of $\mathrm{Sn}(2-3 \mathrm{~nm})$ using argon plasma based sputtering (NEXDEP 120, 5mTorr pressure). The substrate was annealed at $750^{\circ} \mathrm{C}$ for $20 \mathrm{~min}$ in argon ambient $(100$ $\mathrm{sccm}$ ) to facilitate the diffusion of Sn atoms into the top layer of the substrate, which was gradually brought to room temperature to minimize the stress. Excess Sn was removed from the wafer surface using chemical baths.

The SiSn layer so obtained was analyzed for crystalline quality and Sn content. The Sn content was determined using secondary ion mass spectroscopy (SIMS) (Figure 2(a)), showing $\mathrm{Sn}$ incorporation at the substrate surface. Further, the crystalline quality of the SiSn alloy was verified using a cross-section transmission electron microscopy (TEM) close to the surface. It was seen that even after the diffusion of $\mathrm{Sn}$, the silicon lattice was defect-free (Figure 2(b)). Further, we observed the root mean square (RMS) surface roughness of the sample, after Sn diffusion, to be $3.4 \AA$, which was close to the roughness of the original prime grade silicon wafer. Thus, the diffusion of Sn did not cause cracking or grain formation on the substrate surface. However, diffusion leads to a non-uniform concentration of $\mathrm{Sn}$ in the silicon lattice. This non-uniformity creates a gradient in electrical properties such as mobility and effective mass. However, in case of MOSFETs, the inversion layer and the channel are very close to the semiconductor surface ( $\sim 2 \mathrm{~nm}$ depth). The electrical properties of this part of the lattice determine the performance of the transistor. Therefore, we consider the surface concentration of $\mathrm{Sn}$ in the theoretical calculations.

For MOSFET fabrication, we deposited a $400 \mathrm{~nm}$ thick silicon oxide film, as field oxide, using plasma enhanced chemical vapor deposition (PECVD) and then patterned and etched the oxide film using reactive ion etching (RIE) to

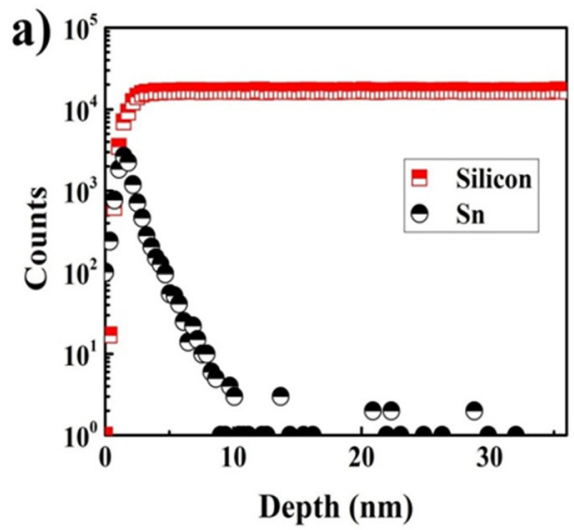

b)

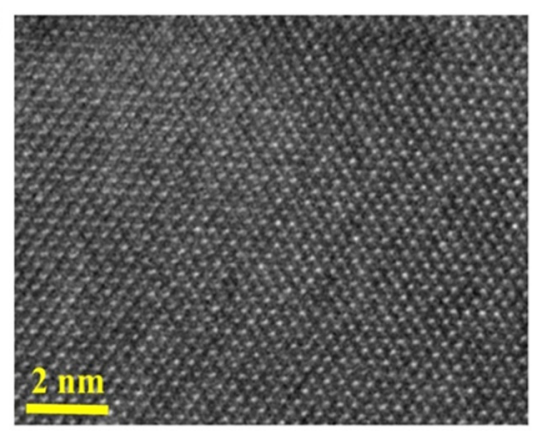

FIG. 2. (a) Secondary ion mass spectroscopy shows the diffusion profile of $\mathrm{Sn}$ in silicon. (b) High resolution TEM image confirms that silicon lattice remains mono-crystalline after diffusion. 


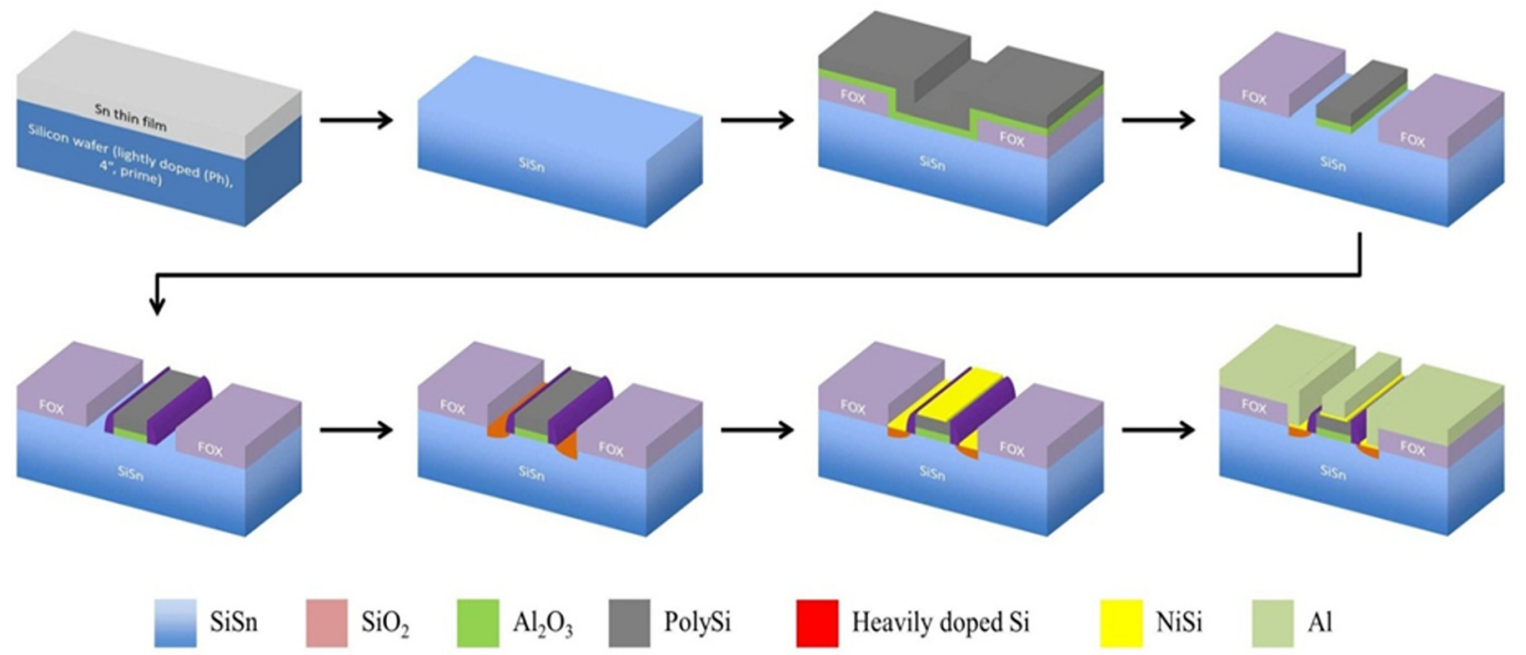

FIG. 3. Schematic illustration of the process flow for device fabrication.

expose the active area. We formed the gate stack by depositing $20 \mathrm{~nm} \mathrm{Al}_{2} \mathrm{O}_{3}$ using plasma assisted atomic layer deposition (ALD), followed by a $200 \mathrm{~nm}$ layer of heavily doped polycrystalline silicon using PECVD $(2-3 \mathrm{~m} \Omega \cdot \mathrm{cm})$. We formed vertical spacers to protect the gate stack from the implantation step by depositing $100 \mathrm{~nm} \mathrm{SiN}_{\mathrm{x}}$ using PECVD, followed by an anisotropic etch using RIE. The source and drain were implanted with boron $\left(\mathrm{BF}_{2}, 10 \mathrm{keV}, 5 \mathrm{E} 15 \mathrm{~cm}^{-2}\right)$ to obtain a heavily doped p-type region. We annealed the wafers at $950{ }^{\circ} \mathrm{C}$ for $30 \mathrm{~s}$ in argon ambient for dopant activation. For formation of ohmic contacts, we salicided the source, drain, and gate regions by depositing $50 \mathrm{~nm}$ nickel using argon plasma based sputtering followed by annealing the substrate in inert ambient at $450{ }^{\circ} \mathrm{C}$ for $30 \mathrm{~s}$. The annealing leads to the formation of nickel silicide and creates an ohmic contact between the contact metal and the heavily doped semiconductor. The unconsumed nickel was removed using a bath of piranha solution for $5 \mathrm{~min}$. We then deposited aluminum as contact metal for probing the devices. Aluminum thin film $(200 \mathrm{~nm})$ was sputtered on the wafer using argon plasma, followed by photoresist spinning and pattering. The aluminum layer was subsequently etched using RIE for metal contact formation. The final devices were sintered at $400{ }^{\circ} \mathrm{C}$ for $5 \mathrm{~min}$ to remove the oxide charges. Figure 3 shows the process flow followed for device fabrication.

\section{RESULTS AND DISCUSSION}

The MOSFET devices were probed for characterization using Cascade Microsystems MA150 probe station. The electrical characterization was done using a Kiethley 4200SCS semiconductor parameter analyzer. To obtain the transfer characteristics of the devices, the source was grounded $\left(V_{S}=0 \mathrm{~V}\right)$, while the gate voltage $\left(V_{G}\right)$ was swept from $+5 \mathrm{~V}$ to $-5 \mathrm{~V}$. For saturation mode of operation, the drain voltage was held at $-5 \mathrm{~V}\left(V_{D S}=V_{D D}\right)$. The transfer characteristics, in saturation mode, for a representative $\mathrm{SiSn}$ and silicon pMOSFET are shown in Figure 4(a).

Since improvement in mobility was expected from the theoretical analysis, we calculated the field effect mobility of SiSn MOSFETs. The field effect mobility is obtained from the transfer characteristics of a device in linear mode of operation. The drain voltage was held at $-50 \mathrm{mV}\left(V_{D S}=V_{D D} /\right.$ $100)$ to obtain the linear mode transfer characteristics. In general, the drain current of a MOSFET is a combination of the drift current and the diffusion current ${ }^{26}$

$$
\mathrm{I}_{\mathrm{D}}=\frac{\mathrm{W} \mu \mathrm{Q}_{\mathrm{n}} \mathrm{V}_{\mathrm{DS}}}{\mathrm{L}}-\mathrm{W} \mu\left(\frac{\mathrm{kT}}{\mathrm{q}}\right) \frac{\mathrm{dQ}_{\mathrm{n}}}{\mathrm{dx}},
$$

where $L$ and $W$ are the length and width of the transistor channel, $Q_{n}$ is the mobile charge density in the channel, $\mu$ is the channel mobility, $k$ is the Bolztmann's constant, $T$ is the a)

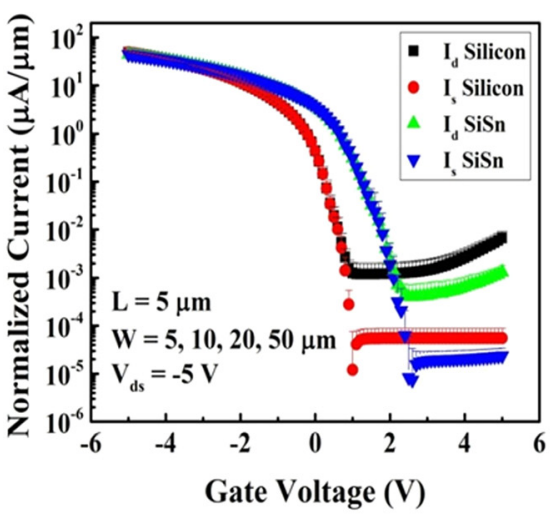

b)

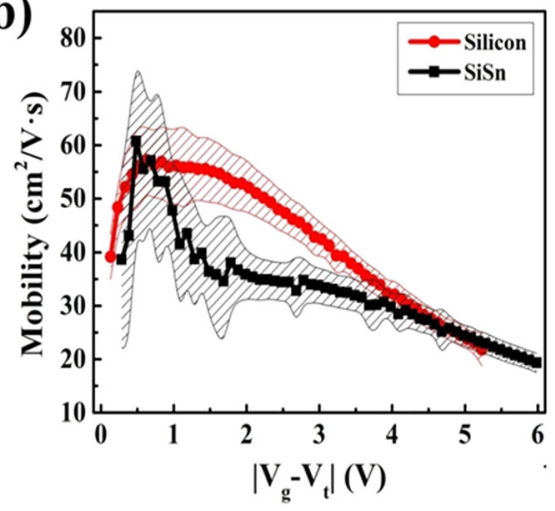

FIG. 4. (a) Transfer characteristics for representative $\mathrm{SiSn}$ devices show better switching characteristics and lower off current compared to the silicon control devices. The symbols represent the average values, while the error bars represent the standard deviations. (b) Field effect mobility for SiSn devices with respect to the gate overdrive. The shaded region represents standard deviation. 
temperature, $q$ is the electronic charge, and $x$ is the distance from the source to the drain. For low channel fields $\left(V_{d s}=-50 \mathrm{mV}\right)$, the channel charge density is mostly uniform from source to drain

$$
\frac{\mathrm{dQ}_{\mathrm{n}}}{\mathrm{dx}}=0
$$

and hence, the diffusion component of the current can be dropped. Further, the charge density in the channel can be approximated as the inversion charge, which is the surface charge that forms due to a strongly negative gate bias that attracts the minority carriers (holes). This inversion charge layer is formed for gate voltages that exceed a certain voltage called the threshold voltage $\left(V_{t}\right)$. This is the voltage at which the transistor is said to have switched, and is obtained by extrapolating the linear part of the transfer characteristics up to the gate voltage axis. In case of inversion mode, the gate acts as a capacitance with the gate oxide as the dielectric and the difference between the threshold voltage and the applied gate voltage (called the gate overdrive), as the voltage between the plates. Hence, the surface charge density is given by

$$
\mathrm{Q}_{\mathrm{n}}=\mathrm{C}_{\mathrm{ox}}\left(\mathrm{V}_{\mathrm{GS}}-\mathrm{V}_{\mathrm{t}}\right)
$$

where $C_{o x}$ is the gate oxide capacitance per unit area. With these assumptions, Eq. (1) is solved for mobility to obtain ${ }^{26}$

$$
\mu_{\mathrm{FE}}=\left(\frac{\mathrm{L}}{\mathrm{WC}_{\mathrm{ox}} \mathrm{V}_{\mathrm{DS}}}\right) \frac{\delta \mathrm{I}_{\mathrm{d}}}{\delta \mathrm{V}_{\mathrm{GS}}} .
$$

The field effect mobility with respect to the applied gate overdrive is shown in Figure 4(b). It is observed that the mobility drops significantly for $V_{G S}=V_{t}$. This is mainly because - as the gate voltage is reduced - the channel charge density reduces, making the ionized impurity scattering and the Coulomb scattering from oxide trapped charges significant. With the increase in gate voltage, the increase in inversion charge screens these scattering mechanisms and phonon scattering dominates, which increases the mobility. However, with further increase in gate voltage, the mobility starts to decrease again due to high phonon scattering. It was observed that the decrease in mobility after the peak value is higher for SiSn compared to silicon. This can be attributed to the fact that phonon scattering is larger in the SiSn lattice due to the presence of large $\mathrm{Sn}$ atoms. The value of effective mobility is taken at the peak of the mobility variation with gate voltage. Figure 5 shows the statistical distribution of the field effect hole mobility for all the characterized devices. We report the average field effect hole mobility for SiSn to be $(75 \pm 9) \mathrm{cm}^{2} / \mathrm{V} \cdot \mathrm{s}$ compared to $(66 \pm 9) \mathrm{cm}^{2} / \mathrm{V} \cdot \mathrm{s}$ for silicon devices. Hence, we have an increase of $13.6 \%$ in the average field effect mobility of the SiSn devices compared to the silicon control. In practice, this increase in mobility can partly be due to the straining of the silicon lattice. Strain engineering in silicon is generally done using epitaxially grown $\mathrm{SiC}$ and $\mathrm{SiGe}$ in the source and drain regions. ${ }^{27,28}$ Hence, diffusing $\mathrm{Sn}$ into silicon can also offer a unique way to strain the silicon channel directly without changing its semiconducting properties.

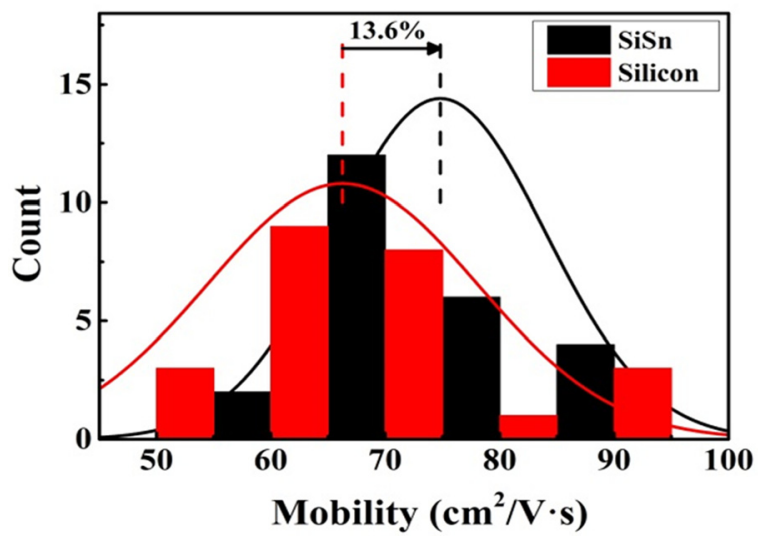

FIG. 5. Statistical distribution of field effect mobility for all SiSn and silicon devices. There is an improvement of $13.6 \%$ in field effect hole mobility after incorporation of $\mathrm{Sn}$ in the silicon lattice.

Therefore, the SiSn MOSFETs were found to have better hole mobility with compared to silicon counterparts. Further, as we reported in our previous work, the off current was found to be lower for SiSn MOSFETs. ${ }^{21}$ The lower effective mass of the holes in SiSn predicted an improvement in mobility. This result has been experimentally verified by fabrication and characterization of MOSFETs, marking a significant advancement in the field of semiconductor electronics, since the performance of silicon p-channel MOSFET has always been lower than that of n-channel MOSFETs, due to the lower hole mobility. ${ }^{29}$ Our work provides an opportunity to increase the mobility of the silicon lattice using a simple diffusion process. Further, implantation of Sn can also be considered as a viable option for obtaining SiSn. ${ }^{30,31}$ Thus, higher mobility SiSn p-channel MOSFETs can be seamlessly integrated in the current CMOS baseline by introducing one additional module.

\section{CONCLUSION}

We present a low-cost process to improve the performance of silicon based logic gates using high performance p-channel SiSn MOSFETs. Standard CMOS fabrication processes have been followed in manufacturing of the $\mathrm{SiSn}$ devices, starting from Sn diffusion into silicon (100). Thus, these devices are fully compatible with the current CMOS technology. We obtained a higher average field effect hole mobility and steeper sub-threshold slope for the devices. The off current was also found to be lower than silicon devices. Hence, the incorporation of SiSn devices into CMOS circuits can yield faster circuits that consume lower power.

\section{ACKNOWLEDGMENTS}

Research reported in this publication was supported by the KAUST Office of Competitive Research Grant No. CRG 1-2012-HUS-008.

${ }^{1}$ L. B. Kish, AIP Conf. Proc. 665(1), 469 (2003).

${ }^{2}$ M. Bohr, in Tech. Dig. - Int. Electron Devices Meet. 2011, 1.1.1-1.1.6.

${ }^{3}$ A. M. Hussain, G. A. T. Sevilla, K. R. Rader, and M. M. Hussain, in Saudi International Electronics, Communications and Photonics Conference (SIECPC) (IEEE, Riyadh, Saudi Arabia, 2013), pp. 1-5. 
${ }^{4}$ K. S. Novoselov, A. K. Geim, S. V. Morozov, D. Jiang, Y. Zhang, S. V. Dubonos, I. V. Grigorieva, and A. A. Firsov, Science 306(5696), 666 (2004).

${ }^{5}$ B. Radisavljevic, A. Radenovic, J. Brivio, V. Giacometti, and A. Kis, Nat. Nanotechnol. 6(3), 147 (2011).

${ }^{6}$ A. Nainani, B. R. Bennett, J. B. Boos, M. G. Ancona, and K. C. Saraswat, J. Appl. Phys. 111(10), 103706 (2012).

${ }^{7}$ R. E. Jones, S. X. Li, E. E. Haller, H. C. M. van Genuchten, K. M. Yu, J. W. Ager, Z. Liliental-Weber, W. Walukiewicz, H. Lu, and W. J. Schaff, Appl. Phys. Lett. 90(16), 162103 (2007).

${ }^{8}$ S. Rajan, S. P. DenBaars, U. K. Mishra, H. Xing, and D. Jena, Appl. Phys. Lett. 88(4), 042103 (2006).

${ }^{9}$ A. N. Hanna, M. T. Ghoneim, R. R. Bahabry, A. M. Hussain, and M. M. Hussain, Appl. Phys. Lett. 103(22), 224101 (2013).

${ }^{10}$ S. J. Wind, J. Appenzeller, R. Martel, V. Derycke, and Ph. Avouris, Appl. Phys. Lett. 80(20), 3817 (2002).

${ }^{11}$ N. Amrane, S. A. Abderrahmane, and H. Aourag, Infrared Phys. Technol. 36(5), 843 (1995).

${ }^{12}$ A. Zaoui, M. Ferhat, M. Certier, B. Khelifa, and H. Aourag, Infrared Phys. Technol. 37(4), 483 (1996).

${ }^{13}$ V. S. J. Rasmus, G. P. Thomas, and N. L. Arne, J. Phys.: Condens. Matter 23(34), 345501 (2011).

${ }^{14}$ J. Franco, B. Kaczer, J. Mitard, M. T.-Luque, F. Crupi, G. Eneman, P. J. Roussel, T. Grasser, M. Cho, T. Kauerauf, L. Witters, G. Hellings, L. Ragnarsson, N. Horiguchi, M. Heyns, and G. Groeseneken, in IEEE International Conference on IC Design \& Technology (ICICDT), 2012 , Austin, USA (IEEE, 2012), pp. 1-4.

${ }^{15}$ H. Genquan, S. Shaojian, W. Lanxiang, W. Wei, G. Xiao, Y. Yue, Ivana, G. Pengfei, G. Cheng, Z. Guangze, P. Jisheng, Z. Zheng, X. Chunlai, C. Buwen, and Y. Y.-Chia, Tech. Dig. Pap. Symp. VLSI Technol. 2012, 97-98.

${ }^{16}$ S. Gupta, B. Vincent, D. H. C. Lin, M. Gunji, A. Firrincieli, F. Gencarelli, B. Magyari-Kope, B. Yang, B. Douhard, J. Delmotte, A. Franquet, M Caymax, J. Dekoster, Y. Nishi, and K. C. Saraswat, Tech. Dig. Pap. Symp. VLSI Technol. 2012, 95-96.
${ }^{17}$ C.-C. Li, K.-S. C.-Liao, C.-H. Fu, T.-H. Tzeng, C.-C. Lu, H.-Z. Hong, T.-C. Chen, T.-K. Wang, W.-F. Tsai, and C.-F. Ai, Solid-State Electron. 78, 17 (2012).

${ }^{18}$ S. Gupta, R. Chen, B. M.-Kope, H. Lin, Y. Bin, A. Nainani, Y. Nishi, J. S. Harris, and K. C. Saraswat, in Tech. Dig. Int. Electron Devices Meet. 2011, 16.6.1-16.6.4.

${ }^{19}$ A. M. Hussain, H. M. Fahad, N. Singh, G. A. T. Sevilla, U. Schwingenschlögl, and M. M. Hussain, Phys. Status Solidi (RRL) 8, 332 (2014).

${ }^{20}$ A. M. Hussain, H. M. Fahad, N. Singh, G. A. Torres Sevilla, U. Schwingenschlögl, and M. M. Hussain, in IEEE 8th Nanotechnology Materials and Devices Conference (NMDC), 2013, Tainan, Taiwan (IEEE, 2013), pp. 13-15.

${ }^{21}$ A. M. Hussain, H. M. Fahad, N. Singh, K. R. Rader, G. A. T. Sevilla, U. Schwingenschlögl, and M. M. Hussain, in 71st Annual Device Research Conference (DRC), Notre Dame, USA (IEEE, 2013), pp. 93-94.

${ }^{22}$ F. Tran and P. Blaha, Phys. Rev. Lett. 102(22), 226401 (2009).

${ }^{23}$ D. J. Singh, Phys. Rev. B 82(20), 205102 (2010).

${ }^{24}$ A. R. Denton and N. W. Ashcroft, Phys. Rev. A 43(6), 3161 (1991).

${ }^{25} \mathrm{P}$. Y. Yu and M. Cardona, Fundamentals of Semiconductors: Physics and Materials Properties (Springer, 2005), p. 206.

${ }^{26}$ D. K. Schroder, Semiconductor Material and Device Characterization (John Wiley and Sons, 2006) p. 489.

${ }^{27}$ T. Ghani, M. Armstrong, C. Auth, M. Bost, P. Charvat, G. Glass, T. Hoffmann, K. Johnson, C. Kenyon, J. Klaus, B. McIntyre, K. Mistry, A. Murthy, J. Sandford, M. Silberstein, S. Sivakumar, P. Smith, K. Zawadzki, S. Thompson, and M. Bohr, Tech. Dig. - Int. Electron Devices Meet. 2003, 11.6.1-11.6.3

${ }^{28}$ Y.-C. Yeo, ECS Trans. 3(7), 1143 (2006).

${ }^{29}$ M. L. Lee, E. A. Fitzgerald, M. T. Bulsara, M. T. Currie, and A. Lochtefeld, J. Appl. Phys. 97(1), 011101 (2005)

${ }^{30}$ S. Fatima, J. Wong-Leung, J. F. Gerald, and C. Jagadish, Appl. Phys. Lett. 74(8), 1141 (1999).

${ }^{31}$ J. Wong-Leung, S. Fatima, C. Jagadish, J. D. Fitz Gerald, C. T. Chou, J. Zou, and D. J. H. Cockayne, J. Appl. Phys. 88(3), 1312 (2000). 